\title{
Carnets
}

Revue électronique d'études françaises de l'APEF

Deuxième série - 7| 2016

Plurilinguisme et migrations dans la littérature de langue française

\section{Plurilinguisme et échange enrichissant dans les deux versions - arabe et française - de Junun de Jalila Baccar}

\section{Chiara Lusetti}

\section{OpenEdition}

\section{Journals}

Édition électronique

URL : http://journals.openedition.org/carnets/1700

DOI : $10.4000 /$ carnets. 1700

ISSN : 1646-7698

Éditeur

APEF

Référence électronique

Chiara Lusetti, «Plurilinguisme et échange enrichissant dans les deux versions - arabe et française de Junun de Jalila Baccar », Carnets [En ligne], Deuxième série - 7 | 2016, mis en ligne le 31 mai 2016, consulté le 30 avril 2019. URL : http://journals.openedition.org/carnets/1700 ; DOI : 10.4000/ carnets. 1700

Ce document a été généré automatiquement le 30 avril 2019.

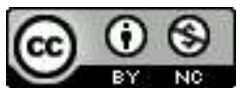

Carnets est mis à disposition selon les termes de la licence Creative Commons - Atribution - Pas d'utilisation commerciale 4.0 International. 


\title{
Plurilinguisme et échange enrichissant dans les deux versions - arabe et française - de Junun de Jalila Baccar
}

\author{
Chiara Lusetti
}

1 Dans cet article, nous explorerons les conséquences de la coprésence de langues différentes à travers l'étude d'un cas d'autotraduction de l'arabe vers le français : la pièce théâtrale Junun de la Tunisienne Jalila Baccar, dramaturge et actrice très célèbre en Tunisie et ailleurs.

2 Nous analyserons une pièce qui nous semble très intéressante pour plusieurs raisons. Elle est tout d'abord remarquable d'un point de vue linguistique, qui sera l'objet de notre étude. Ensuite, elle est fascinante quant aux sujets traités, qui, comme nous le décrirons très rapidement, concernent la société tunisienne dans son noyau le plus intime - la famille - et qui sont considérés comme des thèmes tabous et très violents. Enfin - mais nous ne pourrons pas nous arrêter sur cet aspect dans cet article - elle est considérable pour sa mise en scène, car les réalisations théâtrales de la compagnie dont Jalila Baccar fait partie sont très célèbres au niveau international grâce à leur expérimentation d'avant-garde.

Dans une première partie introductive, nous présenterons la pièce, en résumant sa thématique et en présentant ses personnages. Nous analyserons ensuite les caractéristiques linguistiques générales des deux versions de la pièce, en décrivant comment elles reflètent la situation sociolinguistique tunisienne.

Dans la deuxième partie, nous montrerons l'échange enrichissant qui a lieu, à notre avis, entre les deux langues en présence dans le processus même de la création artistique, soulignant le lien étroit qui unit l'arabe, ou mieux les arabes, et le français dans l'esprit de l'auteure. Nous commencerons par l'analyse du texte arabe, qui est un texte plurilingue, en décrivant le rôle qu'y joue la langue française à la fois de façon explicite, dans des passages en français, et de façon implicite, en influençant la syntaxe de la langue arabe. 
Enfin, nous passerons à l'examen du deuxième texte, en analysant de quelle manière l'arabe suggestionne la langue française, sur le plan lexical et syntaxique.

\section{Jalila Baccar, Junun et les langues en Tunisie}

5 Jalila Baccar, avec son mari et metteur en scène Fadhel Jaïbi, est l'une des figures les plus originales et innovatrices du théâtre tunisien et arabe plus en général ${ }^{1}$. La pièce Junun amène le spectateur à l'intérieur d'une famille tunisienne dont l'un des fils est un jeune prétendu schizophrène. Tout au long de la représentation, le spectateur suit le travail d'une psychothérapeute, qui essaie de le soigner. Mais le spectateur comprend bientôt que les relations de pouvoir et les conflits intimes au sein de la cellule familiale sont les protagonistes réels de la pièce. Tous les personnages portent le nom d'une lettre de

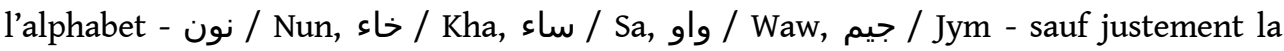
psychothérapeute qui est désignée par le pronom personnel sujet à la troisième personne, الأم Hiya) en arabe, Elle en français, et la mère du protagoniste, qui est appelée هي (alum) / la mère. En résumant, Hiya découvre progressivement que c'est la famille de Nun qui est à l'origine de sa maladie et de la dissociation de sa personnalité. L'accent est mis aussi sur la rébellion de la psychothérapeute contre les méthodes de la psychiatrie traditionnelle et sur sa tentative de soigner Nun en travaillant chez lui, en contact avec sa famille, et non pas à l'hôpital.

6 La pièce a été jouée en arabe pour la première fois en 2001 et publiée en français en 2003 dans une version «Adapté[e] de l'arabe tunisien par Jalila Baccar et Fadhel Jaïbi »². Nous analysons en conséquence ici deux versions de la même pièce, dont l'une écrite dans la langue maternelle de l'auteure et l'autre dans la langue de l'ancien colonisateur.

7 Plus précisément, la pièce en arabe est fortement plurilingue. Dans ce texte, les dialogues se déroulent principalement dans la variété tunisienne de l'arabe, la langue la plus parlée dans le pays. Cependant, dans certains passages c'est l'arabe standard et parfois le français qui sont utilisés. En effet, la structure linguistique de la pièce reflète, d'une certaine manière, la situation sociolinguistique du pays, que nous décrirons brièvement.

8 La Tunisie demeure un espace privilégié pour les pratiques plurilingues : l'arabe standard - langue officielle du pays -, l'arabe tunisien - langue maternelle du peuple et langue de la vie quotidienne -, et le français - langue institutionnelle et véhiculaire - continuent à coexister sur le même territoire (Marzouki, 2014). Cependant, ils ne jouissent pas, comme on le sait, du même statut ni du même capital symbolique ${ }^{3}$. Dans le domaine littéraire, l'arabe standard et le français sont les seuls admis car ils sont regardés comme des langues savantes, bien qu'ils ne véhiculent pas du tout les mêmes représentations : la littérature en arabe standard fait en effet référence au marché littéraire arabophone et à l'idéologie politique du panarabisme ${ }^{4}$, tandis que la littérature en français, langue des colonisateurs et par conséquent des élites, fait un clin d'œil au marché littéraire européen. Au contraire, l'arabe tunisien est souvent considéré comme indigne du milieu littéraire, comme une langue populaire et vulgaire qui ne peut pas exprimer une pensée complexe et de haut niveau intellectuel et artistique ${ }^{5}$. En décrivant ce contexte par les catégories du sociolinguiste Louis-Jean Calvet, nous pourrions dire que ces langues se trouvent en effet dans une relation asymétrique : le français se situe sans aucun doute près du centre dans la galaxie des langues mondiales, tandis que l'arabe reste proche de ses périphéries, et encore plus sa variété tunisienne (Calvet, 1999). Toutefois, ces trois langues continuent chaque jour à s'alterner et à se mélanger dans la vie sociale et 
culturelle tunisienne (Ruocco, 1990 : 272). Du moment que le théâtre de Jalila Baccar se propose de représenter la société tunisienne et en même temps de s'adresser à un vaste public, la dramaturge est obligée de créer ses textes en mélangeant les trois langues, comme nous allons le montrer par la suite, bien que l'arabe tunisien soit prévalent ${ }^{6}$. Dans l'analyse de la version arabe, nous allons nous concentrer dans cet article sur l'usage de la langue française. Malheureusement nous n'aurons pas la possibilité de nous arrêter aussi sur l'usage de l'arabe standard dans la pièce. Toutefois, nous nous proposons d'étudier cet aspect dans nos recherches futures.

9 Si l'on veut envisager le processus autotraductif selon une optique contextuelle, on se trouve ici en présence d'une «autotraduction verticale", c'est à dire d'une autotraduction qui part d'une langue périphérique - et caractérisée par un capital symbolique très réduit -, pour arriver à une langue centrale et prestigieuse. De plus, Jalila Baccar constitue un exemple de "autrotraductrice frontalière », qui n'a pas choisi d'adopter une deuxième langue après une migration ou un déplacement dans l'espace, mais au contraire qui a grandi dans un contexte déjà bilingue, voire trilingue, où le plurilinguisme est un phénomène bien répandu (Grutman, 2009).

10 Après cette brève présentation de la pièce et du contexte linguistique où elle a été créée, nous allons maintenant nous concentrer sur les aspects linguistiques du texte, et notamment sur le rapport entre les deux langues dans les deux versions.

\section{La langue française dans le texte arabe}

Quant à l'analyse du rôle de la langue française dans le texte arabe, nous considérerons tout d'abord sa présence explicite, en soulignant les domaines dans lesquels elle est employée et ses usages spécifiques; nous montrerons ensuite deux phrases en arabe dont la syntaxe ne suit pas les structures habituelles, mais semble influencée par la syntaxe du français.

12 La langue française dans le texte de Junun est souvent employée dans des domaines spécifiques et assez bien reconnaissables, et revient dans toute la pièce. Elle est en premier lieu utilisée dans le domaine de la médicine, qui est évidemment central vu le sujet de la pièce. Nous avons trouvé dans le texte plusieurs occurrences du mot technique «syphilis», nom de la maladie qui affecte Nun à un moment donné, comme dans l'exemple qui suit ${ }^{7}$ :

\begin{tabular}{|c|c|c|}
\hline \multirow{8}{*}{1.} & : يبرى واو : & Waw : Tu crois qu'il va s'en sortir? \\
\hline & الـ: من هي syphilis & Elle : De la syphilis? \\
\hline & $(\ldots)$ & $(\ldots)$ \\
\hline & : من الهبال ساء : & Sa : De la folie \\
\hline & مرضته تعدي & C'est contagieux? \\
\hline & : الهبال هي & Elle : La folie? \\
\hline & الـ: ساء & Sa : La syphilis \\
\hline & (Baccar, $2001:$ 45-46) & (Baccar, $2003: 24-25)$ \\
\hline
\end{tabular}




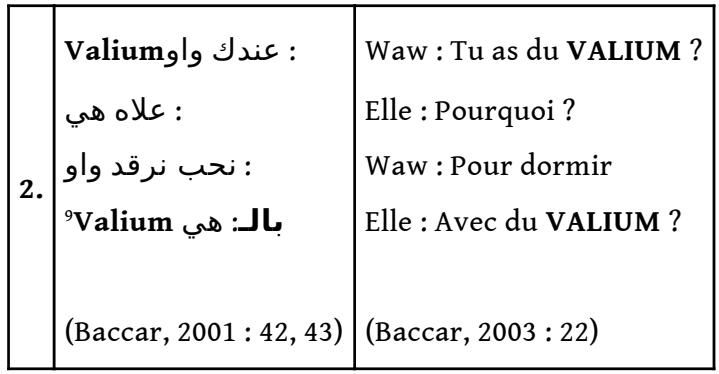

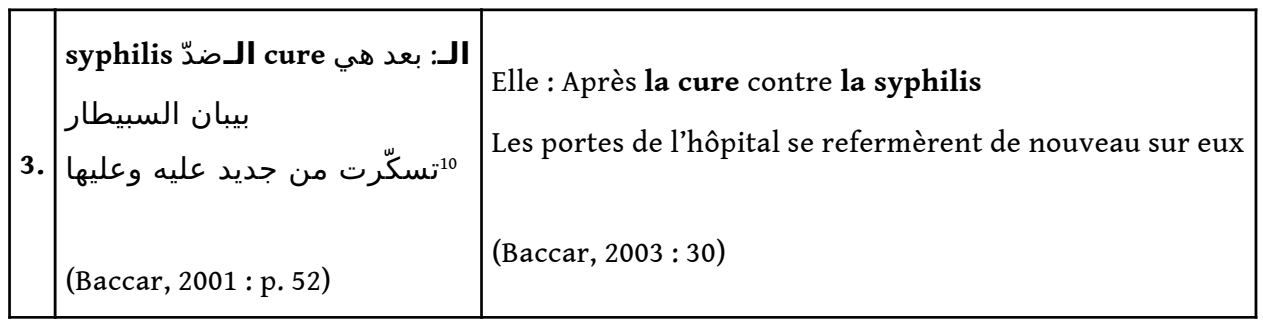

15 Ces mots constituent des emprunts, et il est important de souligner qu'ils sont perçus comme les seuls existants. Cependant leur altérité par rapport à la langue arabe est claire et elle est marquée visuellement par l'usage des lettres latines, insérées directement dans le texte écrit en arabe.

16 Ensuite, nous avons noté dans le texte plusieurs expressions colloquiales françaises qui sont tout à fait acceptables dans l'arabe quotidien tunisien. Nous en présentons ici quelques exemples. Dans l'extrait suivant, l'emprunt correspond à un groupe nominal $[\operatorname{Adj}+\mathrm{N}]$, « Beau Gosse », qui est utilisé comme une sorte de formule :

\begin{tabular}{|l|l|l|}
\hline 4. عزوزة راك نون: & Nun : Tu n'es qu'une vieille peau, tu sais \\
4زوزة وتدلف & Une vieille peau qui fait la fine bouche \\
(Baccar, 2001:140) & Avec un jeune beau gosse comme moi \\
& (Baccar, 2003:107) \\
\hline
\end{tabular}

En revanche, dans l'exemple 5, l'emprunt correspond à une phrase copulative dont le sujet humain est représenté par le clitique « il » et l'attribut par l'adjectif «foutu » selon la structure [Nhum Vêtre + Adj] :

\begin{tabular}{|l|l|l|}
\hline 5. على خاطر كان يقعد لهنا جيم: & $\begin{array}{l}\text { Jym : Car s'il reste dans ce trou... } \\
\text { (Ball est foutu } \\
\text { (Baccar, 2001:50) }\end{array}$ & $\begin{array}{l}\text { Il est fou...tu } \\
\text { (Baccar, 2003 : 28) }\end{array}$ \\
\hline
\end{tabular}


Les deux exemples suivants, enfin, proposent des structures introduites par le présentatif «c'est » suivi d'un adjectif [(C'est) Adj]. Dans l'exemple 6 nous remarquons l'ellipse de la séquence « c'est », qui régit l'adjectif « normal ».

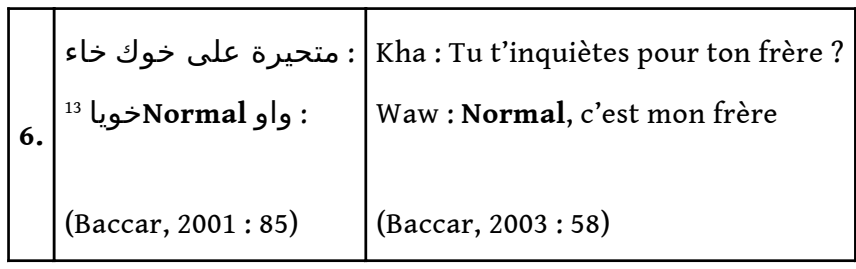

Dans l'exemple 7, en revanche, l'auteure emploie l'expression « C'est bien », dans laquelle le présentatif est suivi d'un adjectif invariable. Cette formule est figée dans l'usage et est utilisée pour exprimer l'approbation. Dans la deuxième occurrence, l'adjectif «bien » est modifié par l'adverbe « très » et le présentatif est à nouveau omis.

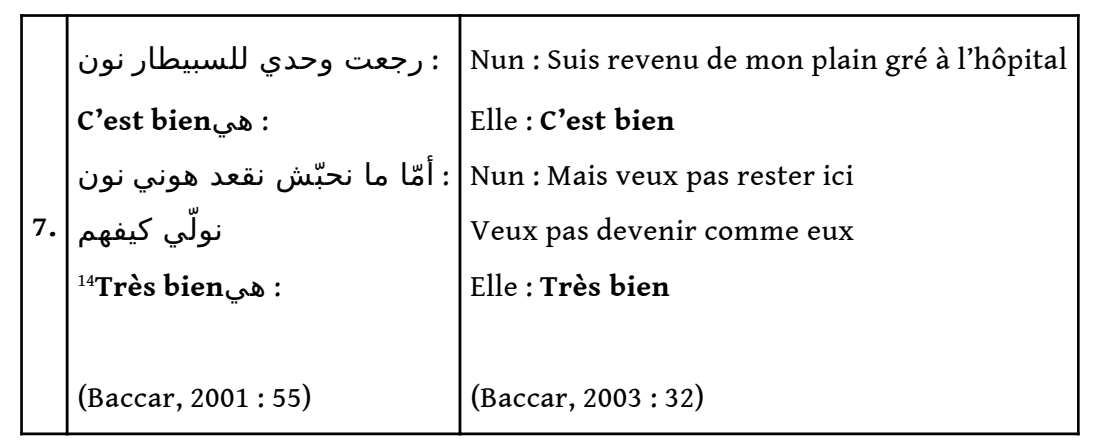

Il s'agit toujours d'expressions qui auraient un équivalent tunisien mais qui sont très utilisées dans la vie quotidienne et qui ne sont pas considérées comme totalement étrangères. En ce qui concerne la syntaxe, il nous semble intéressant de souligner que, malgré les différences en termes de structure que nous avons indiquées, ces expressions sont insérées dans le texte arabe comme si elles formaient des blocs aux éléments inséparables. En effet dans la version française toutes ces expressions sont gardées telles quelles, à l'exception du changement prosodique dans l'exemple $5^{15}$. Jalila Baccar se limite ici, selon notre lecture, à reproduire l'usage linguistique des tunisiens, sans aucun but révolutionnaire ou subversif.

21 Par contre, certains passages du texte révèlent un usage du français très conscient, ayant un but clairement stylistique : la langue française est employée à plusieurs reprises pour mieux définir les personnages et notamment les différences culturelles et sociales entre eux. Dans l'extrait qui suit, Jalila Baccar crée un dialogue entre Hiya, la psy, et Nun, le malade, où l'usage de la langue est fondamental afin de rendre plus clairs les rapports entre les deux personnages. 


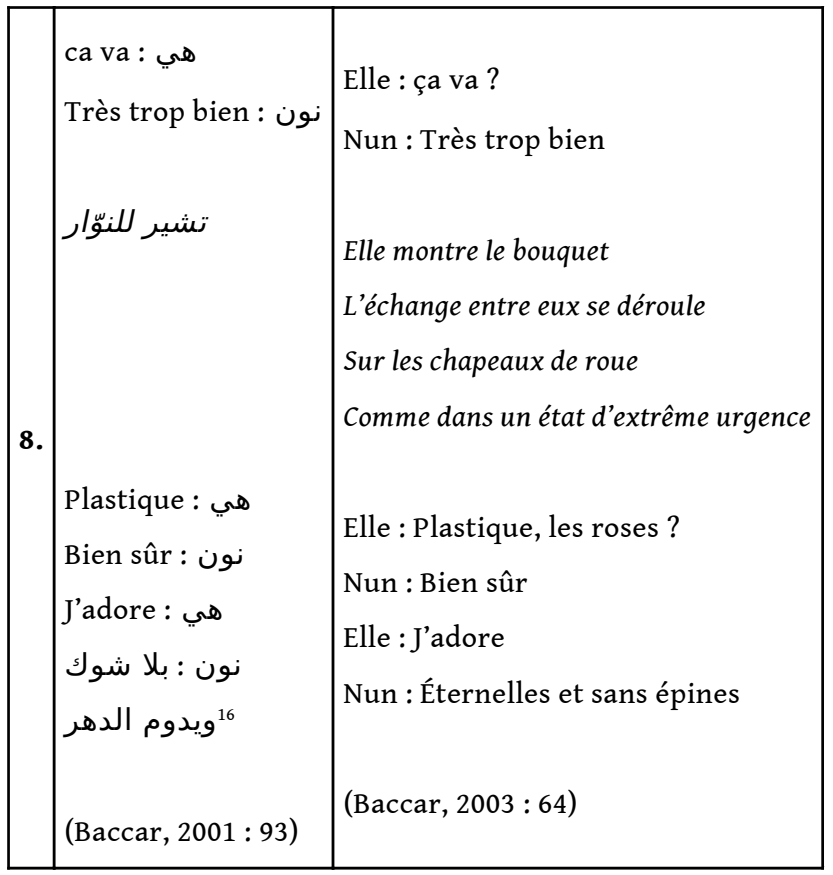

Dans cet échange, Hiya commence à parler en français, en choisissant de dire "ça va », et Nun cherche à la suivre dans ce choix. Nous assistons ici à une négociation de la langue de communication, dans laquelle Nun n'est pas totalement à l'aise. En effet, il répond à Hiya dans un français qui n'est pas correct : l'expression « très trop bien » - qui, comme nous le remarquerons, revient aussi dans le texte français - semble une bonne réalisation de la difficulté que rencontrent plusieurs arabophones dans l'usage de "très » et de "trop ".

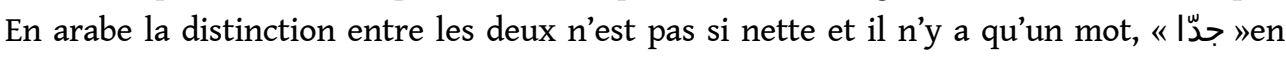
standard et "برشا»en tunisien pour exprimer les deux ${ }^{17}$. L'expression "très trop bien " nous semble une élaboration linguistique exemplaire de cette confusion. Le dialogue continue toutefois en français pendant quelques répliques, mais du coup c'est Nun qui revient à l'arabe, ce qui confirme son état d'insécurité linguistique par rapport à la langue française. Dans l'autotraduction française, Jalila Baccar garde l'échange presque tel quel, en ne corrigeant que la graphie de "ça va ", qui en arabe est écrit sans cédille, sans doute pour reproduire une graphie simplifiée assez répandue en Tunisie. Par conséquent, tout cet aspect de négociation se perd, bien que le caractère arabisé du français de Nun dans l'autotraduction permette de continuer à montrer au public une certaine différence de classe sociale entre les deux personnages.

Nous analyserons maintenant deux exemples de calque syntaxique du français dans le texte arabe. Dans le premier exemple, il s'agit d'une phrase construite d'une manière étrange, qui ne suit pas la syntaxe normale de l'arabe tunisien :

\begin{tabular}{|c|c|c|}
\hline 9. & نون : ثمة برشا شيء نحبّ نقول & $\begin{array}{l}\text { Nun : Y a tellement de choses en moi que j'ai envie de dire, } \\
\text { raconter. }\end{array}$ \\
\hline & (Baccar, $2001: 56)$ & (Baccar, $2003: 33$ ) \\
\hline
\end{tabular}


Nun dit "ثمة برشا شيء نحبّ نقول (Il y a beaucoup de choses que je veux dire), tandis que un locuteur tunisien dirait plutôt " عندي برشا ماقول " (J'ai beaucoup de choses à dire) ou à la limite "نحب نقول برشا شيs (Je veux dire beaucoup de choses). Cette structure semble être un calque de la structure syntaxique «il y a ». Malgré quelques modifications au niveau lexical, la version française « $Y$ a tellement de choses en moi que j'ai envie de dire, raconter " demeure effectivement identique au niveau syntaxique. Le lecteur attentif, en lisant ce passage, a l'impression que Jalila Baccar pense en français, et qu'elle l'a écrit en le traduisant dans sa tête automatiquement du français au tunisien.

On peut observer la même tendance dans l'exemple suivant, où néanmoins l'influence du français est plus discrète et pourrait aisément passer inaperçue.

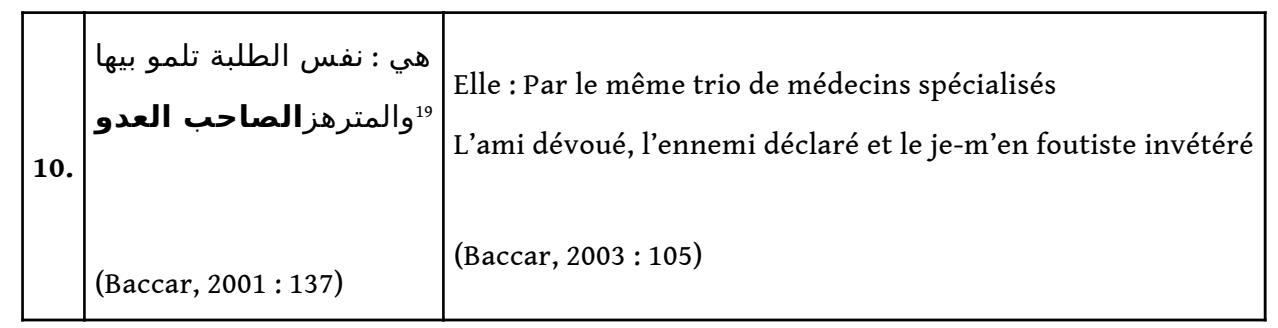

Hiya dans ce passage, comme souvent dans la pièce, se détache de son rôle de personnage pour devenir la voix qui raconte les actions qui ne se déroulent pas sur scène. Elle est en train de décrire le moment où les trois médecins de son équipe lui enlèvent le dossier de Nun à cause de ses méthodes peu orthodoxes. Dans le texte arabe, dans la liste des trois épithètes des trois médecins, il manque le mot $\mathrm{g} /$ waw entre le premier et le deuxième nom, c'est-à-dire la conjonction de coordination qui en arabe est toujours obligatoire et ne peut jamais être omise. Le texte de Baccar dit donc : الصاحب العدو والمترهز "), là où la syntaxe normale de cette phrase devrait être " العدو والمترهزوالصاحر "المند ». Là encore, bien que la différence soit très petite, nous avançons l'hypothèse qu'il ne s'agisse pas d'une erreur, mais au contraire que l'écriture de Jalila Baccar soit influencée par la syntaxe du français, selon laquelle la conjonction de coordination «et» dans une liste est normalement exprimée avant le dernier élément.

27 Nous avons donc analysé deux exemples qui présentent des traces du français dans le texte arabe. Nous allons maintenant choisir et présenter deux exemples de l'influence contraire, de l'arabe sur le texte français.

\section{La langue arabe dans le texte français}

Dans cette dernière partie, nous analyserons, au contraire, deux exemples où l'on peut observer l'influence de l'arabe sur le français. Si, comme nous l'avons remarqué, la pièce arabe est plurilingue, la pièce française, au contraire, est fortement unilingue ${ }^{20}$. Cependant, nous montrerons une certaine influence de la langue arabe sur le texte français. L'arabe est en réalité implicitement présent, bien que de manière beaucoup moins importante par rapport à la langue française dans le texte arabe.

Nous étudierons tout d'abord deux cas de calques lexicaux et après deux cas de calques syntaxiques.

Le premier exemple que nous avons choisi est assez significatif : 


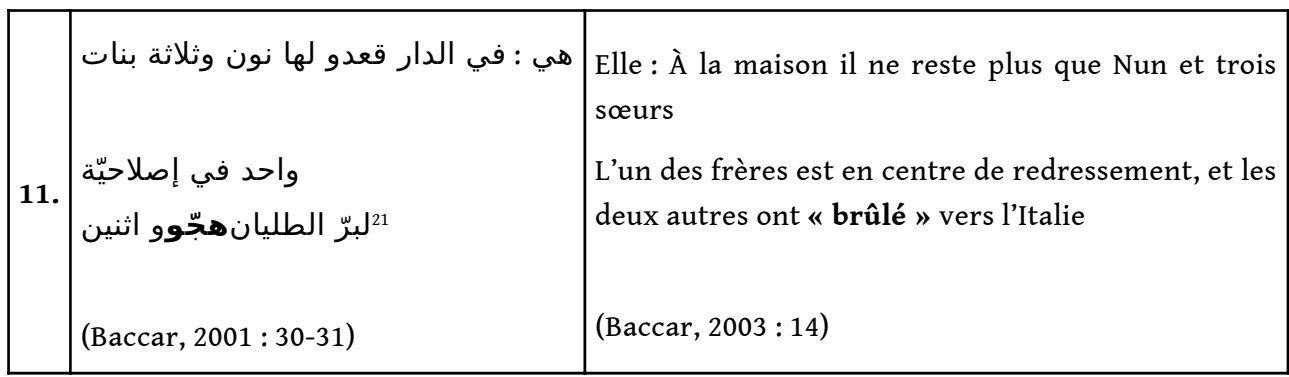

31 À une première lecture du texte français, l'expression «brûler vers l'Italie » paraît, nous semble-t-il, obscure. «Brûler» dans ce contexte constitue un calque du verbe tunisien « ", qui au sens propre signifie justement «brûler» mais qui est utilisé souvent comme un verbe de mouvement, et plus précisément dans le sens de «émigrer vers l'Italie ». Pourtant, il est étonnant de constater que le verbe "حرق" n'est pas présent dans le texte arabe, où au contraire c'est le verbe de l'arabe standard " هرق ه", signifiant tout simplement "émigrer ", qui est utilisé. Nous remarquons ici une trace de sa langue maternelle que Jalila Baccar a laissée dans le texte qu'elle a écrit dans sa deuxième langue. La présence des guillemets laisse sans doute entendre que l'auteure était consciente de l'étrangeté de ce mot par rapport à la langue française et de l'opacité de son sens pour un public francophone, ou bien pour tout public non tunisien.

Ensuite, nous avons choisi un deuxième exemple lexical, mais d'ordre tout à fait différent :

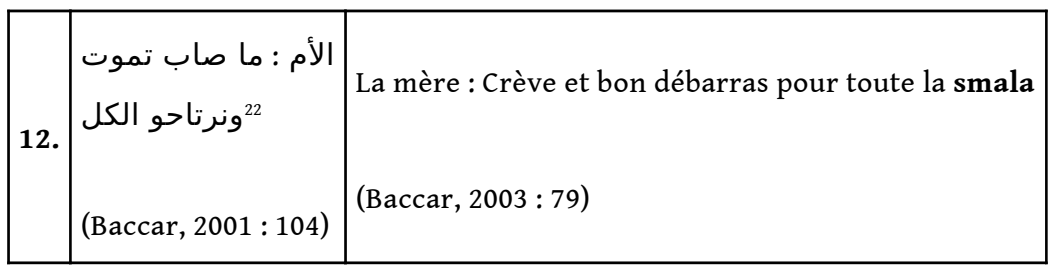

Dans ce passage, Jalila Baccar emploie le nom «smala ", qui est aussi un emprunt de l'arabe et signifie «tribu ». Cependant, il s'agit d'un mot, à l'origine algérien, qui est en train d'entrer progressivement dans la langue française de France, comme le confirme le Petit Robert où nous trouvons cette définition: « Famille ou suite nombreuse qui vit aux côtés de qqn, qui l'accompagne partout > tribu ». L'auteure choisit ici un mot arabe, renvoyant à la réalité maghrébine, qui peut être compris par un bon nombre de français. Elle sacrifie par conséquent la dimension proprement tunisienne du texte, en ouvrant sur un espace plus large, afin de pouvoir s'adresser au public français sans renoncer à l'utilisation de l'arabe.

En revanche, les deux derniers extraits que nous avons choisis concernent la syntaxe : 


\begin{tabular}{|l|l|l|}
\hline 13. & Elle : Ça va ? \\
La mère : Très trop bien \\
(Baccar, 2001:110) & Elle : Qu'est-ce qu'il a ? \\
La mère : Je crois qu'il devient fou \\
(Baccar, 2003: 79)
\end{tabular}

Ici nous avons encore un exemple d'usage arabisé du français. Comme nous l'avons remarqué précédemment, dans l'analyse de l'exemple 8 , les arabophones se trouvent souvent en difficulté dans l'usage de "très » et de «trop ». Nous ne pouvons pas faire ici un parallèle avec le texte arabe, car cette partie est ajoutée dans le texte français. Nous sommes convaincus quand même que ce choix linguistique est très efficace. Jalila Baccar choisit de différencier dans ce passage le langage de la psy de celui de Nun et de sa famille, en rapprochant le français de la mère à l'arabe. De cette façon, elle souligne leur origine sociale plus basse. Cette stratégie, utilisée aussi dans l'exemple 8 avec les mêmes buts, est exploitée à plusieurs reprises, dans le texte arabe comme dans le texte français.

Enfin, nous reprenons l'exemple 5 , que nous avons déjà présenté ci-dessus : nous nous sommes déjà arrêtés sur les parties en français dans la version arabe, maintenant nous allons nous pencher sur la syntaxe de la version française.

\begin{tabular}{|c|c|c|}
\hline 5. & 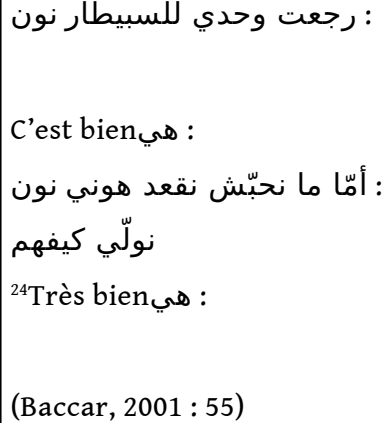 & $\begin{array}{l}\text { Nun : Suis revenu de mon plain gré à l'hôpital } \\
\text { Elle : C'est bien } \\
\text { Nun : Mais veux pas rester ici } \\
\text { Veux pas devenir comme eux } \\
\text { Elle : Très bien } \\
\text { (Baccar, } 2003: 32)\end{array}$ \\
\hline
\end{tabular}

Dans ce passage, Nun efface totalement le pronom personnel sujet « je ». Cet effacement reflète d'un côté la volonté du personnage schizophrène d'anéantir totalement son identité, d'annuler son «Moi »; de l'autre, cette structure nous semble calquée sur la structure arabe. En effet, les répliques en arabe sont ici très courtes, rapides et rythmées. L'omission du pronom personnel sujet en français produit, pareillement, un effet de fragmentation, tout en créant un rythme assez proche du rythme de l'arabe. La syntaxe du français est ainsi déformée pour qu'elle se rapproche du rythme de l'arabe, révélant ainsi un dialogue fructueux et créateur entre les deux langues.

\section{Conclusions}

Les exemples que nous avons choisis et présentés au cours de cette étude mènent à la conclusion que Jalila Baccar, dans le processus de création et d'autotraduction de cette 
pièce qui s'étend dans deux versions, puise dans les deux langues qui, malgré les conflits que nous avons évoqués, constituent deux côtés de son identité. Même si la présence de la langue française dans le texte arabe est beaucoup plus évidente que la présence de la langue arabe dans le texte français, cette différence n'empêche pas de constater l'influence de la langue du peuple tunisien sur la langue des colonisateurs, qui sont aussi les spectateurs potentiels de la version française. Ce dialogue entre les deux versions de la pièce représente, à notre avis, le versant artistique et esthétique du dialogue entre les deux aspects de l'identité linguistique de l'auteure, qui d'un côté incarne une situation assez répandue dans la population tunisienne, mais de l'autre est l'expression d'une réflexion linguistique individuelle très profonde.

\section{BIBLIOGRAPHIE}

BACCAR, Jalila (2001). Junun. Tunis : Sud Edition.

BACCAR, Jalila (2003). Junun, Editions théâtrales. Paris : Passages Francophones.

BALDISSERA, Eros (2004). Dizionario italiano arabo, arabo italiano. Bologna : Zanichelli.

BOYER, Henri (1991). Éléments de sociolinguistique (Langue, Communication et Société). Paris : Dunod.

BOURDIEU, Pierre (1982). Ce que parler veut dire. Paris : Fayard.

CALVET, Louis-Jean (1999). Pour une écologie des langues du monde. Paris : Plon.

CECCHERELLI, Andrea \& Imposti, Gabriella Elina \& Perotto, Monica (2014). Autotraduzione e

riscrittura. Bologna : Bononia University Press.

ECO, Umberto (2014). « Come se si scrivessero due libri diversi » in Ceccherelli (2014).

GRUTMAN, Rainier (2009). «La autotraducción en la galaxia de las lenguas », Quaderns : Revista de

Traducció, 16, Barcellona : Bellaterra, pp. 123-134.

LAGARDE, Christian (2013). L'Autotraduction, aux frontières de la langue et de la culture. Limoges :

Éditions Lambert-Lucas.

MARZOUKI, Afifa (2014). «Plurilinguisme et enjeux identitaires et sociaux dans la Tunisie d'hier et d'aujourd'hui », in Marie-Christine Jullion et Paola Cattani (éds.), Les langues, les cultures et la traduction pour la médiation : perspective d'enseignement et de recherche. Torino-Parigi : L'Harmattan, pp. 39-47.

POULIN, Isabelle (2013). Critique et plurilinguisme. Nîmes : Lucie éditions.

ROBERT, Paul (2007). Le Nouveau Petit Robert. Paris : Le Robert.

Ruocco, Monica (1990). «Lingua fusha o 'ammiyyah nel teatro arabo? L'esempio della Tunisia », Islam. Storia e civiltà $\mathrm{n}^{\circ}$ 9, pp. 271-285.

Ruocco, Monica (2010). Storia del teatro arabo dalla nahda ad oggi. Roma : Carocci.

WEHR, Hans (1979). A dictionary of modern written Arabic. Wiesbaden : Harrassowitz. 
Le Trésor de La Langue Française, version informatique : http://atilf.atilf.fr/tlf.htm

\section{NOTES}

1. Jalila Baccar est née en 1952 à Tunis. C'est une actrice et dramaturge qui a travaillé notamment pour le théâtre, mais aussi pour le cinéma et la télévision. Elle collabore depuis toujours avec son mari, Fadhel Jaïbi, metteur en scène. En 1976 ils ont créé avec d'autres amis et collègues la compagnie المسرح الجديد (Le Nouveau Théâtre), qui s'est efforcée de mettre en rapport le théâtre et le contexte sociopolitique tunisien. Ensuite, en 1993, ils ont fondé la Familia Production, qui a le mérite d'avoir su aborder une sphère tabou de la société arabe : c'est-à-dire la dimension privée de l'existence et le monde des sentiments et des relations familiales, sphère dans laquelle la pièce Junun est totalement plongée (Ruocco, 2010).

2. Il ne s'agirait pas par conséquent d'une vraie autotraduction, mais plutôt d'une adaptation, d'une réécriture à quatre mains, mais vu la solidité de l'union, professionnelle et humaine, entre Baccar et Jaïbi, et le substantiel respect de la structure de l'œuvre originale dans la traduction, nous avons choisi de la considérer comme un cas d'autotraduction. D'ailleurs, plusieurs ouvrages parus dans les dernières années sur le sujet de l'autotraduction montrent de façon assez unanime que les bornes entre autotraduction et réécriture sont en réalité assez floues. Le volume Autotraduzione e riscrittura (Ceccherelli, 2014), qui réunit des contributions assez hétérogènes sur ce sujet, en constitue un bon témoignage. Nous nous limitons à citer un cas exemplaire et influent: le célèbre intellectuel italien Umberto Eco, dans sa contribution, nie presque l'aspect traductif du processus d'autotraduction. En se référant à son expérience d'autotraducteur, il la définit plutôt comme une «reinvenzione in lingue diverse » (Eco, 2014: 27). D'après lui, les différences, les mises à jour, les changements sont inévitables et découlent de plusieurs facteurs, tels que l'évolution de l'esthétique de l'auteur.

3. Nous rappelons que le capital symbolique représente « la reconnaissance, institutionnalisée ou non, qu'ils [les locuteurs] reçoivent d'un groupe » (Bourdieu, 1982 : 68).

4. La langue arabe standard n'est en effet la langue maternelle de personne. Langue de la religion et langue officielle de tous les pays arabes, elle est fortement figée et son évolution est bloquée par des raisons politiques et idéologiques. La dichotomie arabe standard - arabe national est appelée par Louis-Jean Calvet «schizoglossie arabe» (Calvet, 1999: 229), terme que nous préférons à celui plus classique de « diglossie ".

5. Toujours d'après Louis-Jean Calvet, cette situation est fortement antidémocratique, car l'on refuse à un peuple l'accès au savoir dans sa langue. La dévalorisation de la langue maternelle de la majorité de la population amène aussi à une forte insécurité linguistique (Calvet, 1999 : 236).

6. Dans ce contexte, depuis la fin de la colonisation, l'écriture en arabe tunisien représente aussi une prise de position idéologique et politique. Pour un approfondissement de la place de l'arabe tunisien dans le théâtre, voir Ruocco, 1990.

7. Pour chaque exemple que nous allons présenter, nous insérerons à gauche le texte arabe publié en 2001 et à droite le texte français publié en 2003, afin de faciliter la comparaison des deux versions. Les didascalies seront transcrites en italique. Les citations seront numérotées. À chaque fois que nous citerons une partie du texte arabe, nous en proposerons aussi une traduction très littérale en bas de page, pour permettre la compréhension à ceux qui ne connaissent pas cette langue. Notre traduction ne veut absolument pas remplacer la traduction de Jalila Baccar et n'a aucune valeur artistique, mais se propose seulement de rendre le texte accessible aux lecteurs de cette étude.

8. Waw: va-t-il s'en sortir? / Hiya: de la Syphilis? / Sa: de la folie / sa maladie est-elle contagieuse? / Hiya : La folie? / Sa : la syphilis.

9. Waw : t'as du Valium? / Hiya : pourquoi ? / Waw : je veux dormir / Hiya : avec du Valium? 
10. Hiya : après la cure contre la syphilis / les portes de l'hôpital / se fermèrent à nouveau sur lui et sur elle.

11. Nun : t'es vieille / une vieille qui se montre capricieuse / avec un jeune beau gosse comme moi.

12. Jym : parce que s'il reste ici / Il est foutu.

13. Kha : tu t'inquiètes pour ton frère? / Waw : normal, c'est mon frère.

14. Nun : je suis revenu tout seul à l'hôpital / Hiya : c'est bien / Nun : mais je ne veux pas rester ici / devenir comme eux / Hiya : très bien.

15. Nous remarquons que dans la version française Jalila Baccar introduit un jeu phonique qui se base sur les mots fou, tu et foutu, qui riment aussi avec le mot trou à la ligne précédente.

16. Hiya : ça va? / Nun : très trop bien / Elle montre le bouquet / Hiya : plastique ? / Nun : bien sûr / Hiya : j'adore / Nun : sans épines / et ça dure toujours.

17. Voir Baldissera, 2004 ou Wehr, 1979.

18. Nun : il y a beaucoup de choses que je veux vous dire.

19. Elle : Les mêmes médecins se sont rassemblés autour d'elle / l'ami l'ennemi et celui qui s'en fout.

20. La polyphonie linguistique est d'une certaine manière reproduite, à notre avis, grâce à des stratégies différentes, comme par exemple la juxtaposition de registres ou de niveaux de langue hétérogènes. Bien que cela ne soit pas le sujet de cette étude, nous en avons toutefois présenté des exemples : voir les exemples numéro 8 et 13.

21. Elle : À la maison lui restent Nun et trois filles / L'un est dans le centre de redressement / Deux ont migré à l'étranger, en Italie.

22. La mère : Il vaudrait mieux que tu meures et qu'on en finisse tous.

23. Elle : Qu'est-ce qu'il a ? / La mère : on dit qu'il est fou.

24. Nun : je suis revenu tout seul à l'hôpital / Hiya : c'est bien / Nun : mais je ne veux pas rester ici / devenir comme eux / Hiya : très bien.

\section{RÉSUMÉS}

Cet article tente d'explorer les conséquences de la coprésence de langues différentes à travers l'étude d'un cas d'autotraduction de l'arabe vers le français: la pièce théâtrale Junun de la Tunisienne Jalila Baccar. La façon dont l'arabe et le français sont présents dans les deux versions, s'influençant réciproquement de façon créatrice, en dépit des conflits présents dans le contexte, est l'objet de notre article. Nous avons donc mis en évidence, à l'aide de plusieurs exemples, le plurilinguisme du texte arabe et les influences $d u$ français au niveau lexical et syntaxique. Parallèlement, nous avons montré les répercussions de l'arabe sur le texte français, qui est au contraire unilingue. Nous avons ainsi illustré l'échange enrichissant qui se crée non seulement entre les deux langues, mais aussi entre les deux versions de la pièce, qui semblent créer un dialogue entre les deux versants de l'identité linguistique de l'auteure.

This article aims to explore the consequences of the coexistence of different languages throughout a case study of self-translation from Arabic to French: the Tunisian Jalila Baccar's theatrical piece Junun. The subject of this article is the way in which Arabic and French appear in both adaptations, influencing each other creatively, in spite of the conflicts of the context. Therefore, we have attempted to underline the multilingualism shown by the Arabic text and the 
influences exercised by French under a lexical and syntactic point of view, with the aid of numerous examples. Simultaneously, we tried to show the repercussions that Arabic has on the French text which, on the contrary, is monolingual. Therefore, we believe that we have depicted the enriching exchange established not only between the two languages, but also between the two adaptations of the pièce, which seem to produce a dialogue between the two sides of the linguistic identity of the author.

INDEX

Keywords : multilingualism, self-translation, language exchange, French-Arabic translation, Tunisian theatre

Mots-clés : plurilinguisme, autotraduction, échange linguistique, traduction arabe-français, théâtre tunisien

\section{AUTEUR}

\section{CHIARA LUSETTI}

Université de degli Studi di Milano

chiara.lusetti[at]unimi.it 